\title{
Intergalactic Media Densities of Extragalactic Radio Sources and Source Ages
}

\author{
Ezeugo Jeremiah Chukwuemerie \\ Department of Physics and Industrial Physics, Nnamdi Azikiwe University, Awka, Nigeria
}

\section{Email address:}

emailchuksemerie@yahoo.com

\section{To cite this article:}

Ezeugo Jeremiah Chukwuemerie. Intergalactic Media Densities of Extragalactic Radio Sources and Source Ages. American Journal of Astronomy and Astrophysics. Vol. 9, No. 2, 2021, pp. 13-17. doi: 10.11648/j.ajaa.20210902.11

Received: March 16, 2021; Accepted: March 30, 2021; Published: April 20, 2021

\begin{abstract}
In this paper, we use analytical methods with some plausible assumptions to develop a mathematical model which may be used to obtain estimate of density of the intergalactic medium (IGM). This relation suggests that the plot of the linear sizes against the bolometric luminosities of the extragalactic radio sources in our sample should be able to furnish us with an estimate of the density of IGM in which the radio sources are domiciled. Moreover, for the purpose of obtaining an empirical relation similar to the theoretical model, we carry out linear regression analysis of linear sizes and luminosities of the 31 radio galaxies in our sample. The regression result shows an empirical relationship between observed source linear sizes and observed bolometric luminosities with slight correlation whose coefficient is 4 . Moreover, we compared the theoretical relation and the empirical relation to obtain an estimate of the particle number density of the IGM in which the radio sources are located. The estimate indicates a very low density when compared with the values obtained by authors for the compact steep spectrum (CSS) sources. However, while the linear sizes of the CSS sources are of sub-galactic dimensions - they are located within the interstellar media (ISM) - those of the large extended extragalactic radio sources extend into the intergalactic media. The staggering difference in these obtained densities of IGM and ISM simply supports the general notion that there is a sharp decrease in the density at the IGM-ISM interface. Moreover, using theoretical approach again, with some plausible assumptions, we find another relation which may be used to estimate the ages of extragalactic radio sources. This relation possibly indicates that the plot of the linear sizes against velocities of the radio jets of the radio sources in our sample should be able to supply us with an estimate of the sources' ages. We find from the simple linear regression analysis of linear sizes and jet velocities of the radio galaxies in our sample, a relation with correlation coefficient given as 4 , which is also marginal. Comparison of the two relations - theoretical and the empirical relations - gives an estimate of age of the radio sources. The value shows that these large extended extragalactic radio sources are old sources.
\end{abstract}

Keywords: Galactic-intergalactic Interface, Intergalactic Media Densities, Jet Velocity, Linear Size, Luminosity, Interstellar Medium, Radio Sources, Source Ages

\section{Introduction}

Extragalactic radio sources (EGRSs) are those sources with a high ratio of radio to optical emission, commonly defined by the ratio of the two flux densities, $S_{5 \mathrm{GHz}} / S_{6 \times 10^{5} \mathrm{GHz}}>10$ [1]. They are made up of radio galaxies, quasars and BL Lacertae Objects. [2, 3]. The radio emission from these radio sources usually takes the form of relativistic jets that connect the base of the accretion disk to the two radio emitting lobes that straddle the central component that is more or less coincident with the nucleus of the host galaxy. In some radio sources, the lobes contain hotspots believed to be the termination points of the jets [310]. They constitute sources whose radio morphologies range from being compact (e.g. compact steep spectrum sources) to the more extended conventional doubles (e.g. radio galaxies) [7-10].

Presence of jets in radio sources simply suggests presence of gaseous ambient media [4, 7-10]. A number of hydrodynamic simulations of jet propagations have been performed to examine their physical state [4, 5]. These studies show that jet materials have smaller masses than those of the ambient medium. Besides, some authors have created a model for evolution of compact steep spectrum (CSS) sources and used it to estimate their ambient densities 
[6]. Since the linear sizes of these CSS sources lie within the confines of the host galaxies (i.e. $D \leq 20 \mathrm{kpc}$ ) and it is expected that there is a sudden drop of ambient density at the galactic-intergalactic interface, the model needs to be modified to incorporate the more extended sources (i.e. $D \geq 30 \mathrm{kpc}$ ). Therefore, in this work, we modify the model and use it to estimate value of ambient media density and ages of the larger radio sources.

The radio sources used in the analyses are obtained from [11]. They are made up of 31 EGRS (radio galaxies) with linear sizes, $D>80 \mathrm{kpc}$. These sources are those whose jets have estimated velocities from [12].

\section{Radio Source Expansion}

In the standard beam model for extragalactic radio sources, jets of relativistic plasma are ejected from the parent galaxy which plough their way through the ambient medium until they terminate with strong shocks at the hotspots which are thermalized to form lobes [5, 7-10]. The evolution of a radio source component is therefore expected to depend (among other factors) on power supplied by the core to the jet, the source age and the ambient medium through which it propagates.

With an assumption that, in general, jets in EGRSs propagate through a homogenous dense medium, we have [6]

$$
D_{0} \approx\left(\frac{P_{b o l} \mu^{2}}{m_{H} c^{3} n_{e} \Omega \varepsilon}\right)^{0.5}
$$

where $D_{0}$ is intrinsic linear size of the source, $P_{b o l}$ is its bolometric luminosity, $n_{e}$ is its external medium density, $\Omega$ is its jet opening solid angle, $\epsilon(\ll 1)$ is the conversion efficiency of the source for the kinetic power into radiation, $m_{H}$ is proton mass, $c$ is light speed and $\mu$ is the ratio of lobe's velocity to jet's velocity.

The last equation is consistent with a special case in which the angle, $\psi$, between the directions of the velocities, $V_{L}$ and $V_{j}$, of the lobe and jet respectively is $0^{\circ}$ (i.e. for a head-on collision between jet and lobe) which is the case for a homogenous dense medium. However, for a more general case in which $\psi \neq 0^{\circ}$, equation (1) can be modified to become

$$
D_{0} \approx\left(\frac{P_{b o l} \mu^{2} \cos ^{2} \psi}{m_{H} c^{3} n_{e} \Omega \varepsilon}\right)^{0.5}
$$

\section{Orientation Effects on the Observed Linear Size and Unification}

The simplest relativistic beaming and radio source unification model predicts that the observed linear size, $D$, and angle of observation, $\phi$, are related as follows [13]:

$$
D=D_{0} \sin \phi
$$

where $D$ is the observed linear size. Combining equations (2) and (3), we obtain

$$
D \approx\left(\frac{P_{b o l} \mu^{2} \cos ^{2} \psi \sin ^{2} \phi}{m_{H} c^{3} n_{e} \Omega \varepsilon}\right)^{0.5}
$$

The last equation suggestively and generally describes dynamical evolution of extragalactic radio sources with the viewing angle in consideration.

Considering equation (4), it can be seen that the factors which may affect the observed source size, $D$, of a characteristic age, $T$, of a radio source include (i) external medium density, $n_{e}$, (ii) angular difference, $\psi$ between the directions of the velocities, $V_{L}$ and $V_{j}$, of lobe and jet respectively, (iii) angle of observation, $\phi$, and (iv) power of the source or bolometric luminosity, $P_{b o l}$.

We apply some conditions to equation (4) to see how the factors may affect the observed source size, $D$, at a characteristic age, $T$. For simplicity, assuming $\Omega, \varepsilon, \mu$ and $P_{b o l}$ are some constants, then

If $\phi \rightarrow 90^{\circ}, n_{e} \rightarrow 0$ for negligible ambient density, and $\psi \rightarrow 0$ for negligible ambient density, then the result becomes $D \rightarrow \infty$. Therefore, we may observe a large extended radio galaxy.

If $\phi \rightarrow 90^{\circ}, n_{e} \rightarrow \infty$ for an appreciable dense medium, and assuming a homogenous medium in which $\psi \rightarrow 0$, then the result becomes $D \rightarrow 0$. Therefore, we may observe a CSS radio galaxy.

If $\phi \rightarrow 90^{\circ}, n_{e} \rightarrow \infty$ for an appreciable dense medium, and assuming an inhomogenous medium in which $\psi \rightarrow 90^{\circ}$, then the result becomes $D \rightarrow 0$. Therefore, we may observe a compact asymmetrical radio galaxy that could be an asymmetrical CSS radio galaxy.

If $\phi \rightarrow 0, n_{e} \rightarrow 0$ for negligible ambient density, and $\psi \rightarrow 0$ for negligible ambient density, then the result becomes $D \rightarrow 0$. Therefore, we may observe a conventional radio quasar.

If $\phi \rightarrow 0, n_{e} \rightarrow \infty$ for an appreciable dense medium, and assuming a homogenous medium in which $\psi \rightarrow 0$, then the result becomes $D \rightarrow 0$. Therefore, we may observe a CSS radio quasar.

If $\phi \rightarrow 0, n_{e} \rightarrow \infty$ for an appreciable dense medium, and assuming an inhomogenous medium in which $\psi \rightarrow 90^{\circ}$, then the result becomes $D \rightarrow 0$. Therefore, we may observe a morphologically distorted CSS quasar.

We can see from the foregoing that equation (4) supports unification of extragalactic radio sources. This unification simply indicates that the observed characteristics depend on the viewing angle. This may exclude sources with distorted radio morphological structures. In this case, inhomogeneous dense media may have a lot to contribute.

\section{Estimating the Ambient Densities of Larger Radio Sources}

Rewriting equation (4), we have

$$
\left.n_{e} \approx K D^{-2}\right)
$$

where 


$$
K \approx \frac{P_{b o l} \mu^{2} \cos ^{2} \psi \sin ^{2} \phi}{m_{H} c^{3} \Omega \varepsilon}
$$

$K$ can be interpreted to mean a factor for determining ambient density at the galactic-intergalactic interface. Assuming a homogeneous medium in which $\psi$ is $0^{\circ}$, we notice that in the last equation every other parameter except jet opening solid angle, $\Omega$, may have a common value with those of CSS sources (the sizes of CSS sources are of subgalactic dimensions, while those of the larger radio sources are of intergalactic dimensions [7-10]). Moreover, at the galactic-intergalactic interface, it is believed there is a sudden decrease in ambient density; and since gases tend to diffuse faster in a less dense medium than in a denser medium, $\Omega$ is expected to have a larger value at the interface than elsewhere within the host galaxy. For simplicity, we assume a typical value, $\Omega \approx 0.02$ sr [2]; therefore, equation (6) becomes

$$
K \approx \frac{50 P_{b o l} \mu^{2} \sin ^{2} \phi}{m_{H} c^{3} \varepsilon}
$$

However, considering only the intrinsic source size, $D_{0}$, instead of the observed source size, $D$, we have

$$
K \approx \frac{50 P_{b o l} \mu^{2}}{m_{H} c^{3} \varepsilon}
$$

Or with equation (5) for intrinsic source size, we have

$$
n_{e} \approx \frac{50 P_{b o l} \mu^{2}}{m_{H} c^{3} \varepsilon D_{0}^{2}}
$$

Using equation (9) and the typical values of $\varepsilon=5 \%$ [2], $m_{H}=1.67 \times 10^{-27} \mathrm{~kg}, c=3 \times 10^{8} \mathrm{~m} \mathrm{~s}^{-1}, P_{b o l}=P v$ (where $P$ is radio luminosity of each source at frequency of $v=5 \mathrm{GHz}), \mu \approx 0.837[14,15]$, we obtain

$$
D_{0}^{2} \approx\left(\frac{5229.57}{n_{e}}\right) P_{b o l}
$$

The last equation suggests that if a linear regression analysis of the linear sizes $\left(D_{0}\right)$ of the radio sources in our sample against their respective overall luminosities $\left(P_{b o l}\right)$ is carried out, then the gradient of the plot (coupled with the last equation) should be able to furnish us with a rough estimate of the density of intergalactic media in which the radio sources are domiciled.

The radio sources used in the analysis are made up of 31 radio galaxies with linear sizes, $D>80 \mathrm{kpc}$. They were obtained from [11]. These sources are those whose jets have estimated velocities from [13]. We carry out the regression analysis (Figure 1), and obtain the equation given by

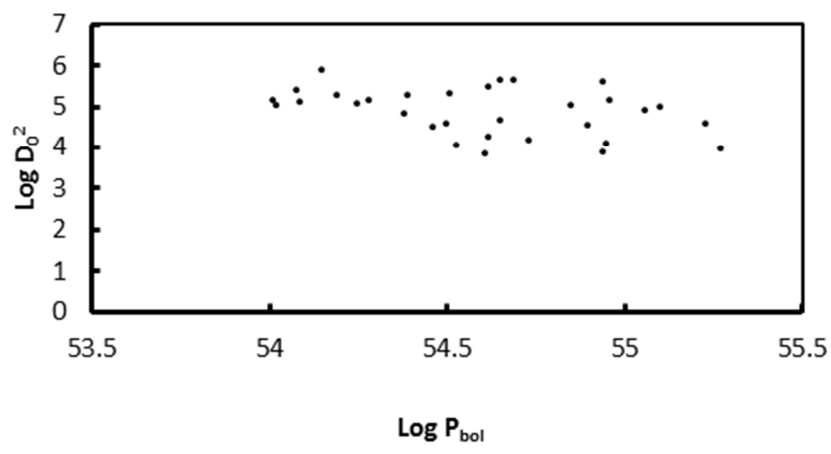

Figure 1. The scatter plot of squared linear size against bolometric luminosity.

$$
\log D_{0}^{2}=-0.63 \log P_{b o l}+39.29
$$

with marginal correlation coefficient, $r=0.4$. Rearranging equation (11), we get

$$
\mathrm{D}_{0}^{2} \approx\left(1.95 \times 10^{39}\right) P_{\text {bol }}^{-1}
$$

which may be referred to as an empirical value for $D-P_{b o l}$ relationship. The index, -1 , which is absent in the theoretical expression (equation (10)) may have been present as a result of marginality in the correlation $(r=0.4)$ of the data. However, we are interested in obtaining a rough estimate the density. Therefore, equating the terms in the brackets of equations (10) and (12), we have

$$
n_{e} \approx 2.68 \times 10^{-36} \text { particles } \mathrm{m}^{-3} \text { ) }
$$

This is a rough estimate of particle number density of the intergalactic media (IGM) in which these radio sources are domiciled. This estimate is by far smaller than those obtained for compact steep spectrum (CSS) sources which are in order of $10^{-11}-10^{-3}$ particles $\mathrm{m}^{-3}$ [6]. The linear sizes of CSS sources are of sub-galactic dimensions, $D_{0}<30 \mathrm{kpc}$ (which is a typical size of a galaxy); while those of the larger radio sources extend into the intergalactic media $[7,9,10]$. The implication of this result is that intergalactic medium is by far less dense than the interstellar medium (ISM) - the medium within the host galaxy. Though, this is a rough estimate, but, it is in consonance with the expected astounding difference in the densities of the IGM and the ISM (i.e. IGM $\leq$ ISM) [2].

\section{Estimating Dynamical Ages of Radio Sources}

The age, $T$, as a function of the intrinsic linear size, $D_{0}$, of a radio source can be expressed as follows [6]:

$$
T=\int_{D_{m}}^{D_{0}} \frac{d D_{0}}{V_{L}}
$$

where $D_{m}$ is the lower limit of the intrinsic linear size and $V_{L}$ is the lobe advance velocity. Combining equations (2) and (14) and putting $D_{m}=0$, yields

$$
T \approx\left(\frac{P_{b o l} \mu^{2} \cos ^{2} \psi}{m_{H} c^{3} n_{e} \Omega \varepsilon V_{L}^{2}}\right)^{0.5}
$$


Assuming homogeneous media, we have

$$
T \approx\left(\frac{P_{b o l}}{m_{H} c^{3} n_{e} \Omega \varepsilon V_{j}^{2}}\right)^{0.5}
$$

Combining equations (10) and (16) to eliminate $n_{e}$, and putting the values of $m_{H}, c, \Omega$, and $\varepsilon$, we obtain

$$
T \approx 2.059 \frac{D_{0}}{V_{j}}
$$

Or, we have

$$
D_{0} \approx(0.486 T) V_{j}
$$

The last equation says that source age can be estimated from linear regression analysis of observed linear sizes of the radio sources against their respective jet velocities. We carry out the analysis (Figure 2) using the aforementioned 31 radio galaxies and obtained

$$
\log D_{0}=0.358 \log V_{j}+19.14
$$

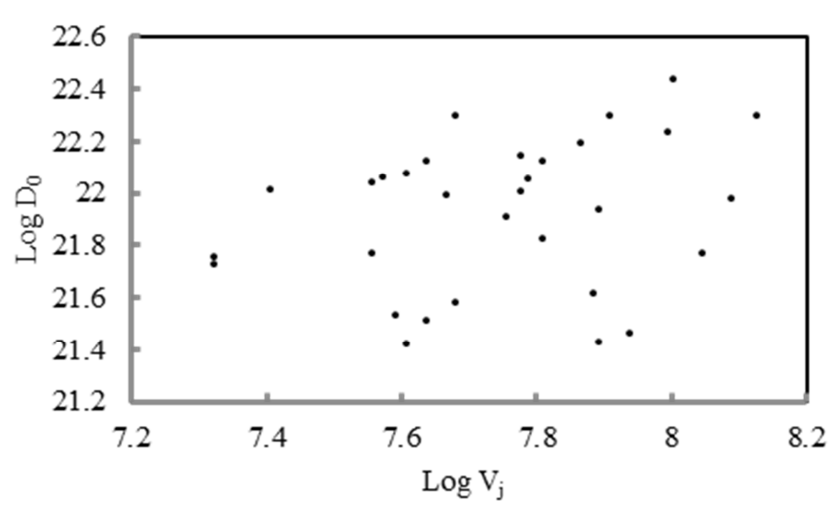

Figure 2. The scatter plot of linear size against jet velocity.

The correlation coefficient, $r \approx 0.4$. This is also marginal. Rearranging the last equation gives

$$
D_{0} \approx\left(1.51 \times 10^{19}\right) V_{j}^{0.4}
$$

The last relation may be referred to as an empirical value for the $D_{0}-V_{j}$ relationship. Just as before, the index, 0.4 , which is absent in the theoretical relation (i.e. equation (18)) may have been present as a result of marginality in the correlation $(r=0.4)$ of the data. However, for now we are interested in obtaining rough estimate of the sources' age. Therefore, equating the terms in the brackets in equations (18) and (20), age of the radio sources is estimated to be in the order of

$$
T \sim 10^{11} \mathrm{Yr}
$$

implying they are old sources.

\section{Discussion of Results}

We have used analytical methods with some plausible assumptions to find a relation (equation (10)) which may be used to obtain estimate of density of the intergalactic medium
(IGM). This relation suggests that the plot of the linear sizes $\left(D_{0}\right)$ against the bolometric luminosities $\left(P_{b o l}\right)$ of the radio sources in our sample should be able to supply us with an estimate of the IGM in which the radio sources reside. We find from the simple linear regression analysis of the 31 radio galaxies in our sample, a relation of the form shown in equation (12); with correlation coefficient, $r \approx 0.4$, which is marginal. We can see that the two expressions (equations (10) and (12) for theoretical and empirical values respectively), are alike, except for the index $(-1)$ which is absent in equation (10). This difference may have resulted from the marginality in the correlation of the data. However, comparison of the two relations gives an estimate of the particle number density of the IGM in which the radio sources are located to be $\approx 2.68 \times 10^{-36}$ particles $\mathrm{m}^{-3}$. This is quite a small value when compared to the values in the range of $10^{-11}-10^{-3}$ particles $\mathrm{m}^{-3}$ obtained by [6] for compact steep spectrum (CSS) sources. These CSS sources are of sub-galactic dimensions $\left(D_{0} \leq 20 \mathrm{kpc}\right)$ - they are located within the interstellar media (ISM). The staggering difference in these densities of IGM and ISM simply supports the general notion that there is a sharp decrease in the density at the IGM-ISM interface [2, 14].

Moreover, using theoretical approach again, with some plausible assumptions, we find another relation (equation (18)) which may be used to estimate the ages of the radio sources. This relation indicates possibly that the plot of the linear sizes $\left(D_{0}\right)$ against velocities of the jets $\left(V_{j}\right)$ of the radio sources in our sample should be able to provide us with an estimate of the sources' ages. We find from the simple linear regression analysis of the radio galaxies in our sample, a relation given as equation (20), with correlation coefficient, $r \approx 0.4$, which is also marginal. It is easily seen that the two expressions (equations (18) and (20) for theoretical and empirical values respectively), are alike, except for the absence of the index (0.4) in equation (18). This difference may have resulted from the marginality in the correlation of the data. However, comparison of the two relations gives an estimate of age of the radio sources. This is given by $T \approx 9.88 \times 10^{11} \mathrm{Yr}$.

\section{Conclusion}

Using both analytical and statistical methods with some plausible assumptions, we have developed mathematical relations for estimating intergalactic media densities and dynamical ages of large extended extragalactic radio sources. Results show that the difference in the densities of intergalactic media and those of the interstellar media is enormously large. We also find those sources to be old.

\section{References}

[1] Urry, C. M., (2004) Unification: An Update. Astronomical Society of the Pacific conference series, 1 , no p.

[2] Robson, I., (1996) Active Galactic Nuclei”. Praxis Publishing Ltd, England. 
[3] Readhead, A. C., (1995) Evolution of Powerful Extragalactic Radio Sources. Proceedings of Colloquuium on Quasars and Active Galactic Nuclei, USA: National Academy of Sciences, Berkman Center, Irvine.

[4] Jackson, J. C. (1999) Radio Source Evolution and Unified Schemes. Publications of Astronomical Society of the Pacific, $16,124-129$.

[5] Kawakatu, N. and Kino, M. (2007) The Velocity of Largescale Jets in a Declining Density Medium. Proceedings of Conference on Triggering Relativistic Jets, 27, 192-197. 2007.

[6] Ezeugo, J. C. and Ubachukwu, A. A. (2010) The Spectral Turnover - Linear Size Relation and the Dynamical Evolution of Compact Steep Spectrum Sources. Monthly Notices of the Royal Astronomical Society. 408, 2256-2260.

[7] Mahatma, V. H., Hardcastle, M. J., Williams, W. L (2019) LoTSS DR1: Double-double Radio Galaxies in the HETDEX field. Astronomy and Astrophysics, 622, A13.

[8] Mingo, B. J., Croston, H., Hardcastle, M. J. (2019) Revisiting the Fanaroff-Riley Dichotomy and Radio Galaxy Morphology with the LOFAR Two-Meter Sky Survey (LoTSS). Monthly Notices of the Royal Astronomical Society, 488, 2701-2721.

[9] Hardcastle, M. J., Williams, W. L., Best, P. N. (2019) Radioloud AGN in the First LoTSS Data Release - The Lifetimes and Environmental impact of Jet-Driven Sources. Astronomy and Astrophysics, 622, A12.

[10] Dabhade, P., Gaikwad, M., Bagchi, J. (2017) Discovery of Giant Radio Galaxies from NVSS: Radio and Infrared Properties. Monthly Notices of the Royal Astronomical Society, 469 (3), 2886-2906.

[11] Nilson, K. (1998) Kinematical Models of Double Radio Sources and the Unified Scheme. Monthly Notices of Royal Astronomical Society, 132.31-37.

[12] O’Dea, C. P., Dally, R. A., Kharb, P. and Freeman, K. A. (2009) Physical Properties of Very Powerful FR II Radio Galaxies. Astronomy and Astrophysics, 494, 471-569.

[13] Ubachukwu, A. A. and Chukwude, A. E. (2002) On the Relativistic Beaming and Orientation Effects in CoreDominated Quasars. Journal of Astrophysics and Astronomy, $23,235-24$.

[14] Ezeugo, J. C. (2015) On Jet Opening Angle and Dynamical Evolution of Some Powerful Extragalactic Radio Sources. American Journal of Astronomy and Astrophysics, 3, 40-43.

[15] Owsianik, I. And Conway, J. E. (1998) First Detection of Hotspot Advance in a CSO - Evidence for a Class of Very Young Extragalactic Radio Sources. Astronomy and Astrophysics, 336, 69-79. 